\title{
El diseño instruccional de los MOOC y el de los nuevos cursos abiertos personalizados ${ }^{1}$
}

\section{Instructional design of MOOCs and the new personalized open online courses}

\author{
Miguel Zapata-Ros \\ Universidad de Murcia. Centro de Formación y Desarrollo Profesional. España. \\ mzapata@um.es
}

\begin{abstract}
Resumen
En este trabajo vamos a exponer resumidamente una propuesta justificada y fundada de un procedimiento para realizar el diseño instruccional de un curso que eventualmente pueda ser un MOOC pero también un curso en línea, abierto y personalizado.

Está organizado en dos partes, la primera es una recensión sobre aspectos teóricos a tener en cuenta en el diseño instruccional de cursos abiertos online, la segunda está constituida por una guía sucinta para el diseño instruccional.

Éste es el comienzo de un trabajo que desarrollaremos de forma completa y detallada en otros trabajos de naturaleza adecuada: En artículos, guías y ejemplos de aplicación a casos.
\end{abstract}

Palabras clave: Diseño instruccional, MOOC, aprendizaje personalizado

\begin{abstract}
This article deals with a justified, sound, summarised proposal for a procedure to create the instructional design of a course. This course might be a MOOC, but also an open, personalised, on-line course.

The article is organised into two sections. The first one is a review of theoretical aspects which should be taken into account for the instructional design of open, online courses. The second one is a brief guide for instructional design.

The review and the guide are the first part of a larger piece of work, which will be developed in full and in a more detailed way in future presentations, such as articles, guides and cases as examples.
\end{abstract}

Key words: Instructional design, MOOC, Personalised learning

\footnotetext{
${ }^{1}$ El presente trabajo ha estado accesible desde el 6 de agosto de 2013, como preprint, en el repositorio eLis. Además ha sido objeto de una sesión en la res social científica Academia.edu que ha durado 30 días hasta el 20 de febrero de 2015. Durante ambos periodos de exposición y debate ha recibido numerosas críticas y comentarios que han dado lugar a modificaciones del texto original. El autor agradece a los colegas que han participado de esta forma en su edición actual su inestimable colaboración y aportaciones.

Por otro lado este trabajo constituye una parte sustancial del material que se está utilizando en el cuso abierto online que se está impartiendo desde la Universidad de Alcalá, en una estructura de tres capas y que tiene como contenido y objetivo capacitar a profesores en el diseño instruccional de cursos abiertos online. Se trata de un cuso investigativo que pretende recoger datos y evidencias sobre los temas que trata y sobre su modalidad de organización en capas y con metodología de Mastery learning, profesorado Teaching assistant y otras innovaciones recogidas en este trabajo. La dirección del curso es https://openeducation.blackboard.com/mooc-catalog/courseDetails/view?course_id=_232_1 y el blog donde se difunden las incidencias http://cursosabiertos.blogspot.com.es/
} 


\section{Introducción.-}

Han cobrado extraordinaria vigencia los debates sobre la naturaleza y sobre las características de un nuevo modelo de enseñanza superior que se vislumbra tras la irrupción de los MOOC, con fuerza primero y de manera crítica después. Ha sido un debate genérico, sobre el nuevo modelo o sobre el modelo en ciernes. Pero también han cobrado importancia los subdebates derivados de él, las proyecciones del debate general sobre otras discusiones: el modelo de negocio, las condicionantes sociales y económicas, el contexto de sociedad en cambio donde se produce, el de los recursos, su naturaleza abierta. Pero más allá de estos debates y del discurso sobre las metodologías docentes emergentes-recurrentes, lo cierto es que hay dos circunstancias innegables en presencia, dos hechos constatados:

El primero lo constituye la evolución de los modelos nacientes de MOOC, o de los cursos en línea en general, hacia opciones que evolucionan muy rápidas. Se han catalogado hasta 10 tipos, según Clark (2013), o un número indefinido según el sistema de 12 dimensiones propuesto por Conole (2013). E incluso de la conversión de estos en modelos nuevos como son los que se enumeran en "Sistemas de Apoyo al Aprendizaje y al Rendimiento" (Zapata-Ros, 2015a) (ver figura 1) con un denominador común: El carácter predominante de la nueva educación universitaria como una educación abierta.

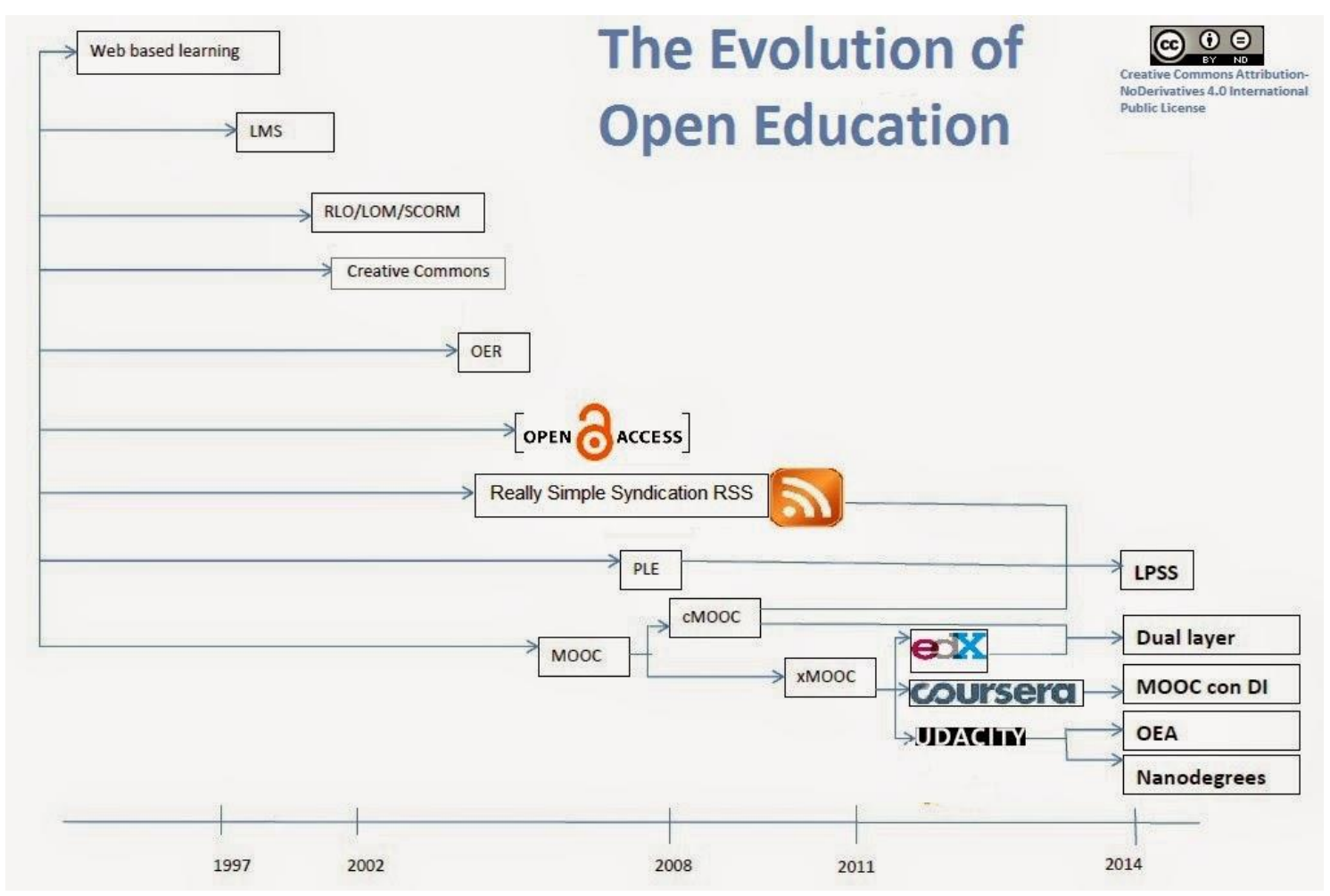

Fig.1 La evolución de la Educación Abierta

El desarrollo de estas líneas va a continuar (Zapata-Ros, 2014a), es la esencia de los xMOOC, que una vez transformados en una modalidad de formación universitaria (en la alianza OAE según hemos visto (Zapata-Ros, 2014a)) el resultado es coherente con lo que Ferenstein (Sep 9, 2013) señala como su objetivo: 
(...) hay un montón de estudiantes brillantes de todo el mundo que sólo tienen acceso a los cursos a través de Udacity o KhanAcademy; la Alianza OEA permitiría a estos prodigios, por primera vez, para demostrar su talento a las empresas de tecnología, tomar cursos y obtener certificados de los proveedores de educación en línea.

\section{(...)La OEA va a crear (esperemos) una nueva meritocracia en la educación superior.}

Pero hay otra línea de desarrollo en el uso de la tecnología como apoyo a la educación, es la línea adaptativa, a la que con más propiedad hemos llamado de personalización ${ }^{2}$. Término que, recordemos, hemos tomado en el sentido de utilizar los recursos que la tecnología nos proporciona para, a través de su socialización, conseguir una percepción lo más completa posible de cuáles son las preferencias y las representaciones que el individuo posee de la realidad y del mundo que le rodea, cuáles son las características de su andamiaje cognitivo, para a partir de él, y de su rol en él, presentar los nuevos conocimientos.

El otro hecho constatado es el cambio sobre su línea inicial de ausencia de diseño instrucional, o si lo queremos el avance desde situaciones casi de espontaneismo pedagógico explícito, defendido en los primitivos cMOOC por los autores ${ }^{3}$ (Downes, 2011 a través deWiley, 2012 y Zapata-Ros, 2013a p.33 y 36), hasta y hacia modelos de diseño instruccional cada vez más apoyados en los avances de la moderna pedagogía, de las teorías del aprendizaje, de los modelos sobre cómo se aprende, se evalúan los aprendizajes y cada vez más apoyado también en el diseño instruccional (Weller, 2013). Tendencia que Coursera pone de manifiesto en su guía Building a Coursera

2 Descartamos la expresión "adaptativo" por dos razones. La primera por diferenciarlo del "aprendizaje adaptativo", suficientemente conocido, que únicamente utiliza las necesidades de aprendizaje del estudiante para presentarle los contenidos de aprendizaje, y en nuestra propuesta lo que se individualiza es el diseño instruccional en su conjunto, o en lo más posible, para adaptarlo a las características de aprendizaje detectadas en el entorno personal del alumno. Y la segunda para hacer más énfasis en el sujeto de la expresión, que es el que tiene que hacer el esfuerzo para adaptarse a una situación compleja y cambiante. El ser humano es, en un plazo más largo que el de su vida, un sistema adaptativo. Pero nosotros hablamos de sistema educativo. En nuestro caso la fuerza recae sobre el objeto del sistema, en este caso el individuo y sus características de aprendizaje.

La expresión adaptativo se vincula con sistema adaptativo complejo (CAS, del inglés complex adaptive system) que es un tipo especial de sistema complejo; El ser humano sí se puede considerar como un sistema complejo, como lo es la bolsa de valores o un ecosistema, en el sentido que se utiliza en estos dominios teóricos. En el sentido de que es diverso y conformado por múltiples elementos interconectados, algunos de los cuales son emergentes.

Pero en este caso los sistemas educativos no hacen énfasis en estos aspectos o en esta dimensión del ser humano por ser una dimensión ontológica que no tiene como referencia el individuo singular. A los sistemas educativos les interesa el individuo singularizado en un intervalo de tiempo que afecta a una fase de su vida o exclusivamente a su vida.

En todo caso cabría hablar de meta-adaptativo pero solo atendiendo a las formación de las competencias que desarrollan las características adaptativas del individuo.

3 One of the philosophical underpinnings of MOOCs as practiced by Siemens, Downes, et al. has been the rejection of the idea of pre-defined learning outcomes. For example, the LAK12 syllabus reads in part:

"You are NOT expected to read and watch everything. Even we, the facilitators, cannot do that. Instead, what you should do is PICK AND CHOOSE content that looks interesting to you and is appropriate for you. If it looks too complicated, don't read it. If it looks boring, move on to the next item.” The learning outcomes will, consequently, "be different for each person." 
Course (CIT, 2013), un manual ortodoxo sobre diseño instruccional, secuenciación, elaboración de guías didácticas, etc. y también en su apuesta por el método de tutoría, evaluación y docencia Mastering learning (Brandman, 2013).

Hay que constatar que en cualquier caso los MOOC, por su naturaleza de acceso indiscriminado, aunque algunos de ellos como veremos sean personalizados, mayoritariamente no es ésta su característica principal. Este hecho afecta a elementos del diseño instruccional como son la definición de objetivos, la evaluación, los recursos o el diseño de actividades. Pero hay un elemento que es inmune a esta naturaleza de los MOOC, y que siempre hay que tener en cuenta, sea cual fuese el grado de apertura de acceso o de personalización. Nos referimos a la secuenciación.

Para todas las modalidades de MOOC o de cursos en línea personalizados los contenidos han de ser secuencializados según las técnicas clásicas, que atribuyen un papel central, en cómo se organizan, a la forma de aprender o de representarse el conocimiento que tiene los alumnos, a sus preferencias, objetivos personales, concepciones, etc. Esto constituye una base común del diseño.

\section{Bases teóricas.-}

Lo primero que nos planteamos cuando iniciamos este trabajo, en consonancia con todo lo anteriormente elaborado, sobre todo cuando hablamos del conectivismo como base pedagógica de los MOOC (Zapata-Ros, 2013a), ha sido la validez y el alcance de las teorías existentes en los nuevos entornos. Teorías universalmente válidas, que se basan en evidencias acerca de las características del aprendizaje. Siempre en la perspectiva de un diseño instruccional centrado en el aprendizaje y por ende en el alumno. Así pues es pertinente preguntarse qué fuentes y que referencias y conocimientos teóricos podemos utilizar. No podemos despreciar, como siempre hemos insistido, todo el bagaje de investigaciones y de evidencias sobre la eficiencia de métodos docentes, de sistemas de evaluación y sobre todo lo que conocemos acerca de cómo los individuos construyen su conocimiento, y los rasgos de éste para que sea un auténtico aprendizaje, es decir que tenga los requisitos de apropiación por el alumno, atribución de sentido y transferencia. O lo que es lo mismo, para que sea eficaz en la práctica de su aplicación, de su transferencia y para que sirva de base a nuevos conocimientos y destrezas. E indagar qué parte de esto sirve para basar el diseño de la instrucción, la definición de objetivos la evaluación, etc.

En el esquema más simple, podemos considerar para la práctica de los sistemas de formación una organización de este tipo que vincule teoría y práctica mediante evaluación formativa y diseño instruccional, de modo que aquella nos suministre elementos para elaboración y redefinición de los principios teóricos, y éste sea el enlace con esos principios para iluminar la práctica en escenarios y contextos concretos: 


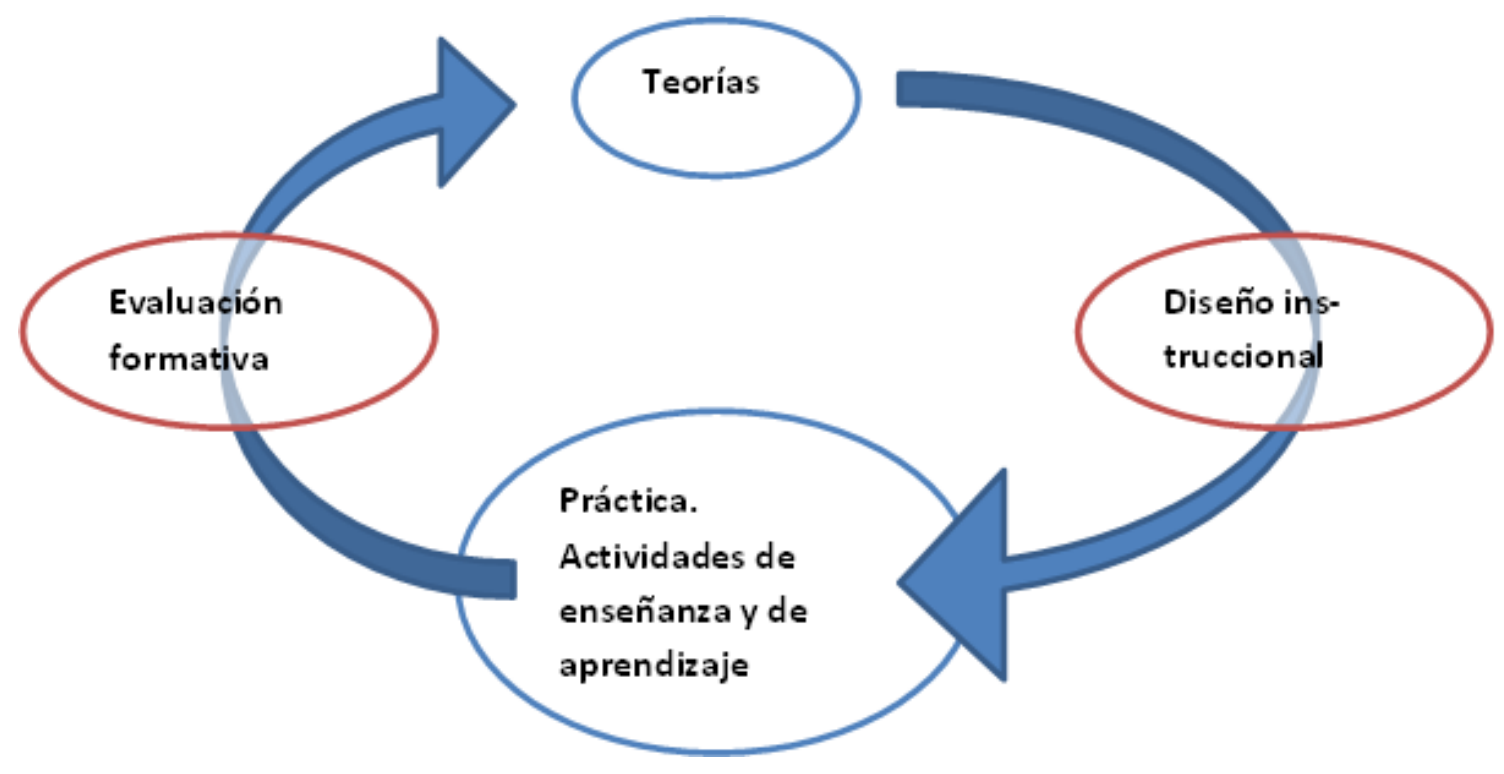

Fig. 2 Fuente: Las teorías del aprendizaje y el diseño instruccional.

Una vez construido este esquema (Fig. 2), con posterioridad, analizando los precedentes que podrían servirnos y entre ellas las valiosas aportaciones de (CIT,2013) y de Fink (2003), que hemos utilizado como base y como referencia para nuestro trabajo, encontramos el esquema de Coursera y el de Fink que lo inspira:

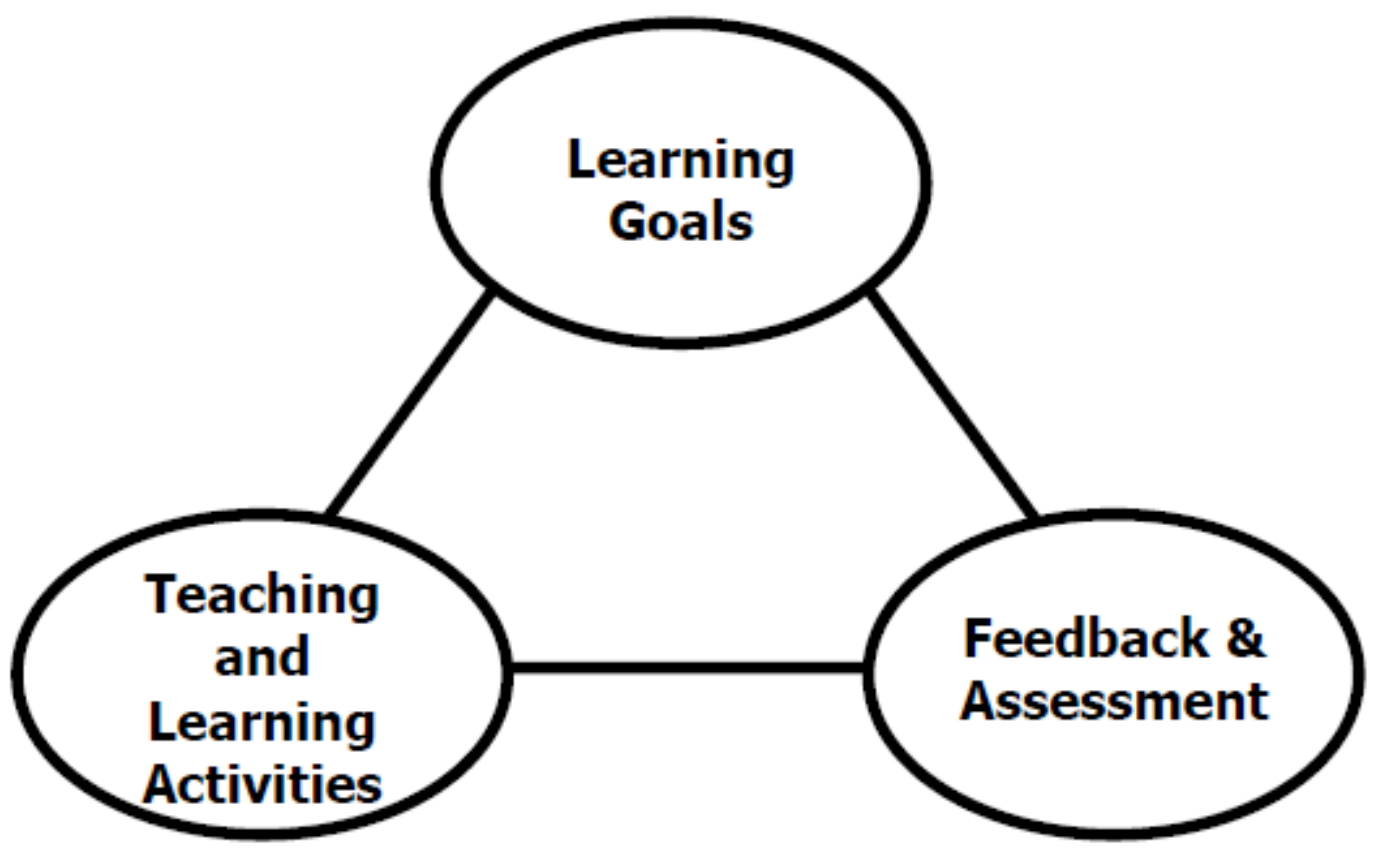

Fig.3 Esquema de Coursera y de Fink “Componentes clave para el diseño de un curso integrado"

Encontramos un innegable paralelismo entre ellos. De esta forma se pueden identificar y sustituir en Fig.2 la "actividades de enseñananza y aprendizaje" por "Práctica. Actividades de enseñananza-aprendizaje", "Objetivos de aprendizaje" por "Diseño instruccional" (más completo y complejo) y "Feedback y evaluación" por "Evaluación formativa". Quedando un esquema similar al anterior (Fig.2). Evidentemente el 
esquema de Coursera-Fink (Fig. 3) resultaría incompleto porque faltan las bases teóricas, las teorías. No creemos que esto sea un olvido o una limitación, simplemente han considerado que la naturaleza práctica de la guía las hacía innecesarias.

De esta forma, en su documento Fink (2013) nos priva de citas y de referencias, sin ser es originales suyas las ideas y sin estar justificados los procedimientos. En la parte que más conocemos, la secuenciación, está tomado todo literalmente de la "Teoría de la elaboración" de Reigeluth y Merrill (Reigeluth, Merrill, Wilson \& Spiller, 1980 (Reigeluth \& Stein, 1983) (Reigeluth, 1999). No nos extraña pues que en la guía nos prive también de las bases teóricas.

De esta forma, sin embargo, en un contexto como el que estamos, de recensión y con una perspectiva investigativa, el esquema sería diferente, sería el de la figura 2 . Y en un contexto de práctica Fink utiliza en sus guías el de la figura 3.

Para el diseño instruccional de cursos abiertos on line es imprescindible pues tener un conocimiento claro del carácter que tienen las teorías y no solamente de los elementos más interesantes para aplicar. De esta forma, de acuerdo con sus rasgos pero no de una forma estricta o alternativa las teorías están enmarcadas en tendencias y hay referencias e influencias inevitables a constructos centrales para el diseño instrucional. Podemos señalar (Zapata-Ros, 2014b) que existe una línea de continuidad en la adaptación de las estrategias docentes, de la ayuda pedagógica, poniendo los bienes del conocimiento cerca de las distintas formas de aprender y de integrar los conocimientos en los esquemas y en las representaciones individuales. Esta línea arranca en los trabajos que hace Reigeluth, que le llevan a formular en sus distintas versiones la Teoría de la Elaboración, y en los trabajos previos sobre el andamiaje cognitivo, la significación de los contenidos de aprendizaje, los esquemas cognitivos, y las distintas teorías de secuenciación que realizaron Ausubel, Gagné, Merril y Novak (Zapata-Ros, 2014b), continúa en la definición de técnicas del diseño instruccional, a la luz de estas teorías, que permiten diseños de programas formativos centrados en los alumnos y en el aprendizaje. Estos como otros constructos operativos y otros que también son operativos los señalamos en el esquema más completo siguiente: 


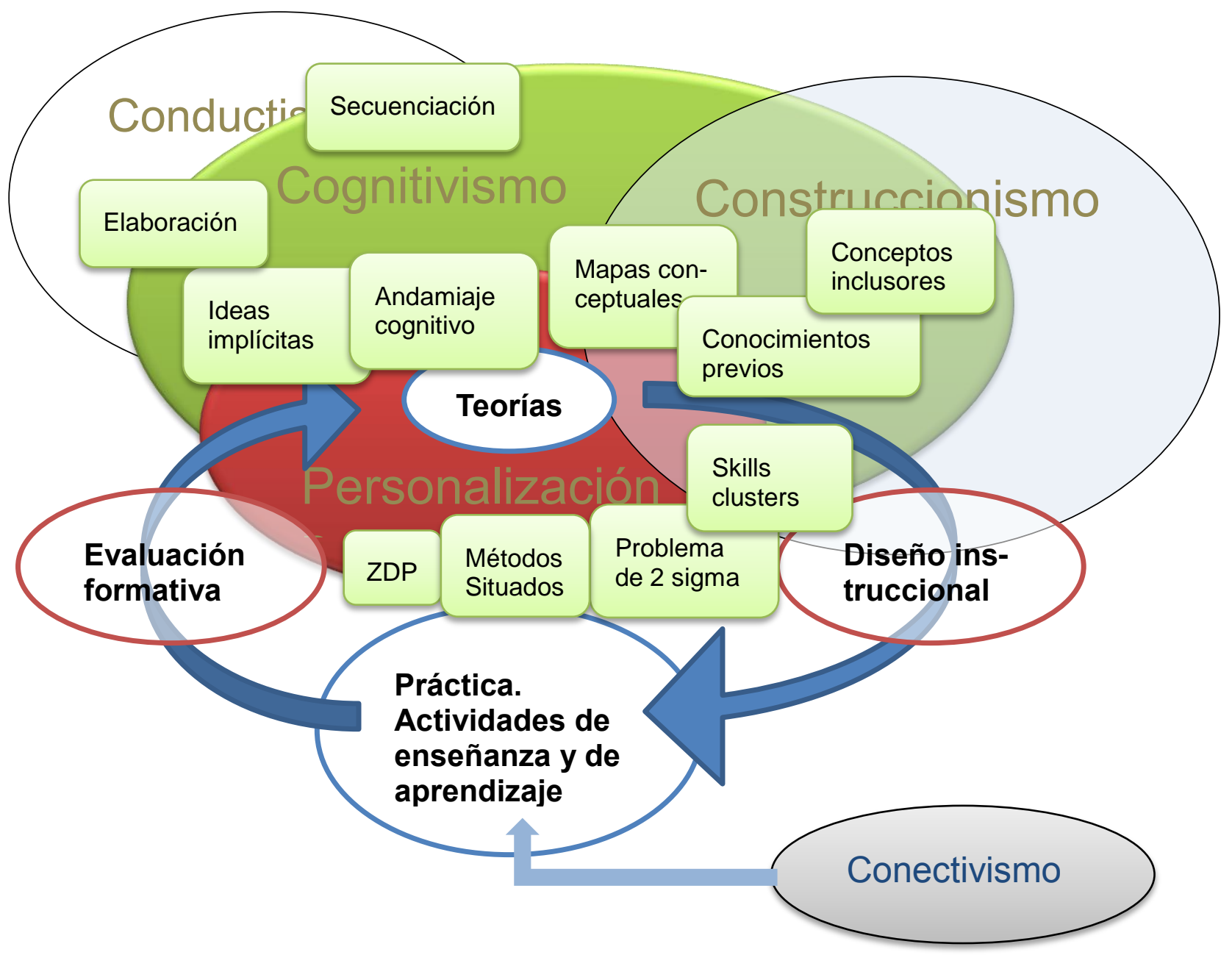

Fig.4 Esquema de teorías que ilustran el diseño instruccional

Alguien podría objetar que situamos al conectivismo, en el esquema, fuera de las teorías del aprendizaje. El ubicar la perspectiva conectivista aparte es consecuencia de nuestro análisis (Zapata-Ros, 2012) de considerar al conectivismo, tal como lo presenta su autor original (Siemens, 2004), sólo como una interpretación de algunos de los procesos que se producen en el seno de la Sociedad de la Información y del Conocimiento, relacionados con la educación, en la que se atribuye un significado y una proyección de estos cambios en el ámbito de la práctica educativa y de su organización. No obstante este corpus de ideas ha tenido y tiene en la actualidad un gran impacto en el mundo académico y en la evolución posterior de los cursos online y de los recursos abiertos hacia los MOOC, de los primero de los cuales, los cMOOC, son el sustento pedagógico.

Cabe decir, en función de todo ello, y en primer lugar de las declaraciones de los propios creadores (Downes, 2011), que el conectivismo es un conjunto de ideas 
conectado directa e implícitamente, como una influencia o una inspiración, sin mediación reflexiva o explícita en el diseño (Zapata-Ros, 2012)

Coherentemente con lo dicho en todo este trabajo hay que señalar, también en relación con el esquema, la importancia que cobran las perspectivas teóricas que tienen que ver con la "personalización del aprendizaje". Y conferir a todo lo relacionado con éste la naturaleza de un nuevo dominio de las teorías del aprendizaje. Un nuevo dominio que engloba aquellas perspectivas clásicas, con las más recientes, y que pueden ser útiles a la hora hacer un diseño nuevo: el que tiene que ver con la adaptación del diseño a los individuos con puntos comunes.

Se está produciendo un tránsito entre dos conceptualizaciones, y las teorías que las sostienen y las justifican. Suponen dos paradigmas en el desarrollo de las teorías educativas y del aprendizaje y en la práctica de la educación: En el primer caso, la perspectiva imperante en la Sociedad Industrial, las teorías y la práctica de la organización educativa están orientadas a describir y a clasificar a los alumnos desde la perspectiva de sus capacidades, en el segundo caso, la perspectiva de la nueva Sociedad Postindustrial, del Conocimiento, tanto las teorías como las prácticas están orientadas a maximizar el aprendizaje de todos los alumnos. Y en cada uno de los casos se hace con distintos valores: En el primero el progreso se mide en función del tiempo (de pautas y ciclos temporales: Cursos, clases, etc.), y en el segundo se basa en los logros individuales (Reigeluth, 2012).

Un rasgo de este cambio es que el aprendizaje es un fenómeno no solamente personalizado sino un fenómeno situado. Por tanto el nuevo diseño instruccional tiene que tener presente aquellas conceptualizaciones. Hay principios que, aun siendo universales, dan lugar a aplicaciones distintas según las situaciones de que se trate. Este hecho tiene otra repercusión: El que los métodos educativos sean situados supone un alto protagonismo de los profesores y de los diseñadores instruccionales. Se introduce un nuevo parámetro: La precisión del método.

De hecho así lo reconoce Fink (2003) en su esquema que sirve de referencia a Coursera (Fig. 5) para su guía para diseñadores intruccionales de MOOCs. 


\section{The Key Components Of INTEGRATED COURSE DESIGN}

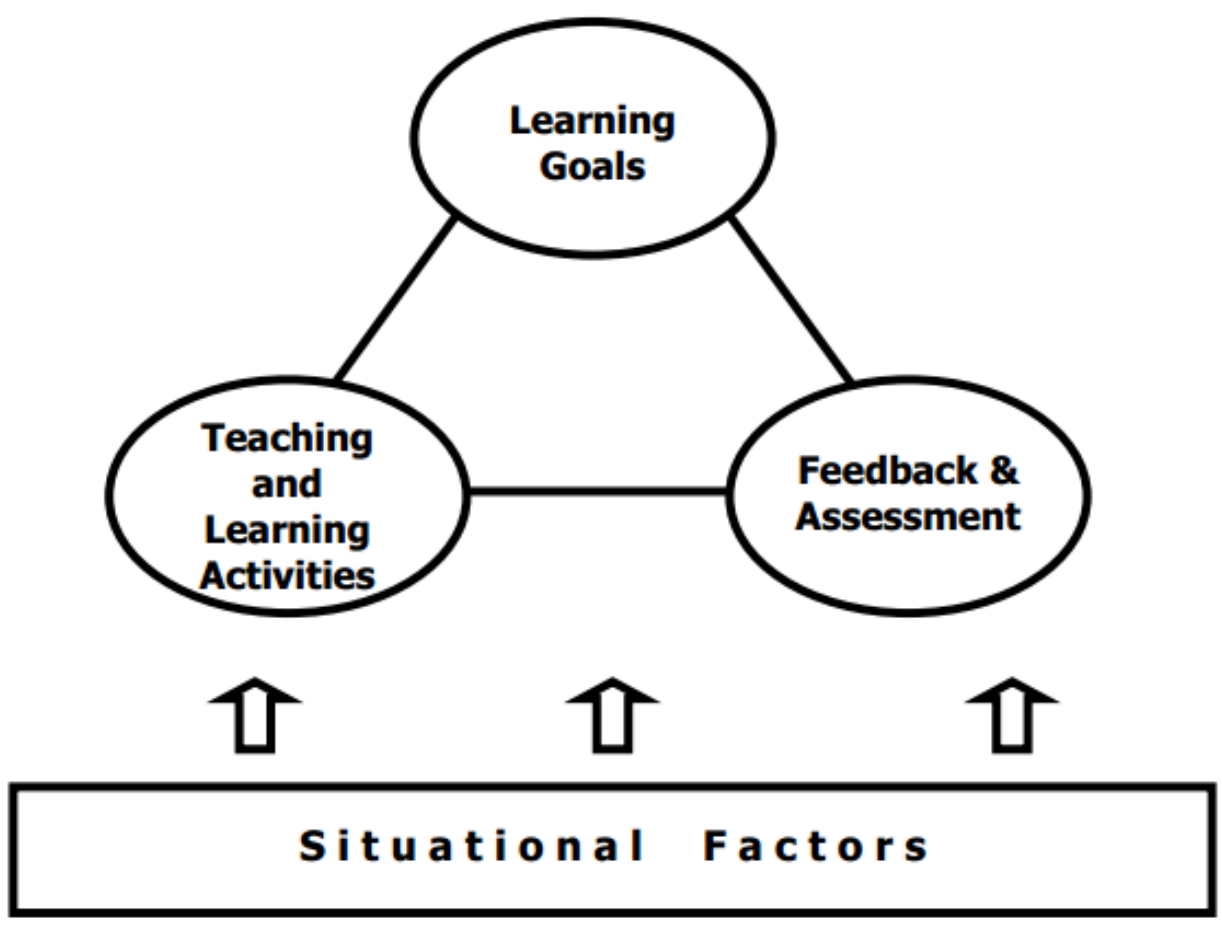

Fig.5 Esquema de Fink ampliado con factores situacionales.

Esto hace que, como establecemos en el esquema (Fig. 4), tengan especial relevancia en esta perspectiva de la personalización del aprendizaje elementos teóricos tales como: Los tradicionales de zona de desarrollo próximo" (ZDP) y los racimos de competencias (skills clusters), o más recientes como los métodos situacionales, el problema de 2 sigmas o los que derivan del pensamiento divergente.

\section{Guía para diseñar un curso que, eventualmente, pueda ser un MOOC pero también un curso en línea, abierto y personalizado.}

Una vez establecidas las coordenadas teóricas del problema, en lo que sigue hacemos una propuesta sucinta de una guía de diseño instruccional para programas de formación abiertos online, guía que adjuntamos íntegra como ANEXO 1 a este trabajo. En este primer acercamiento sólo queremos establecer una relación de los puntos que debe abordar la guía, con una explicación escueta del significada y pertinencia de cda uno de ellos. Corresponderá en cada caso concreto, siguiendo el principio de "principios universales escenarios concretos" propuesta por Reigeluth (2012), desarrollar una guía explícita y desarrollada adecuada al programa de que se trate.

Hacemos esta presentación acogiéndonos a la posibilidad que ofrece RED, Revista de Educación a Distancia, de publicar artículos que contemplen 
- desarrollo y prueba de una o más tecnologías particulares de aprendizaje

- estudios de caso de prácticas innovadoras

En lo sucesivo utilizaremos pues un lenguaje más directo y concreto, más propio de este tipo de documentos, de las guías docentes, que de las reflexiones o de los ensayos teóricos.

En primer lugar es necesario decir que intentamos que nuestra propuesta integre distintas opciones que vayan desde cursos sin prácticamente diseño instruccional como son los MOOC, donde la intervención del profesor instructor es prácticamente inexistente, y el diseño se limita casi exclusivamente a distirbuir una secuencia de contenidos y actividades de forma más o menos laxa a través de un periodo de tiempo a veces ni tan siquiera limitado, juno con propuestas de cursos online convencionales, los que se desarrollan en los LMS convencionales, a las propuestas que constituyen nuestro objetivo preferente que son los cursos abiertos con metodología docente y evaluación de "mastery learning". En particular esta opción de uso en ambientes flexibles se ha concretado en nuestra propuesta (Fig. 6) de curso de tres capas (Zapata-Ros, 2015b) pero que igualmente podría ser útil en cursos del tipo "dual layer" (Fig. 7) (Siemens, 2014 May)(Crosslin, 2014 May). De ahí lo de ambientes flexibles, que van desde el propio y conocido de cursos masivos a cursos "mastery learning" tutorizados.

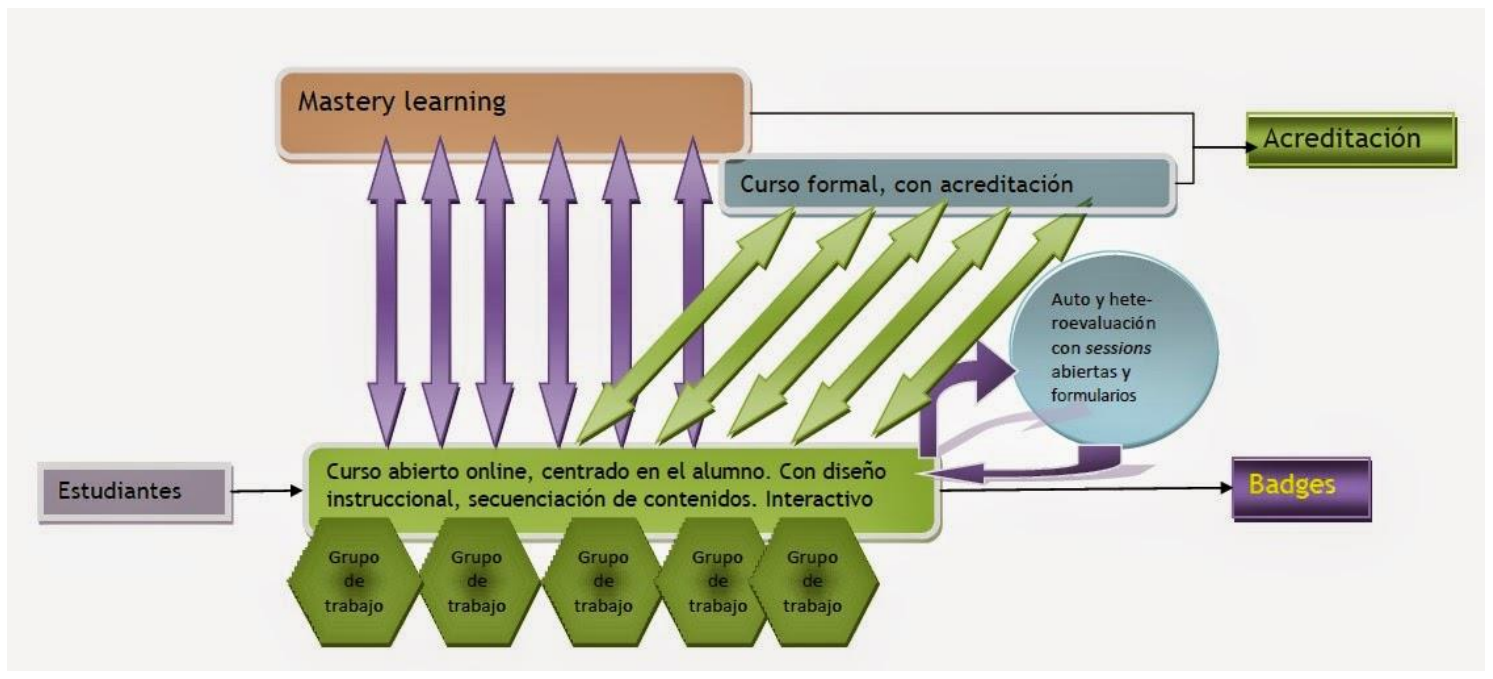

Fig. 6 Curso de tres capas (Zapata-Ros, 2015b) 
DUAL LAYER cMOOC/XMOOC



Fig. 7 Esquema de cuso de dos capas (dual layer) (Siemens, 2014 May)(Crosslin, 2014 May).

Como aspecto central y clave que planteamos, sobre profesores y docencia, es la importancia de tener en cuenta todo el tiempo dos cosas:

1. En los cursos en línea hay un solo tipo de profesor, que tiene naturaleza de instructor. Este profesor y esta naturaleza ha de ser preeminente en todas las funciones sobre cualquier otra.

2. La fase donde más influencia tiene el profesor sobre el aprendizaje es en la fase de diseño, durante la construcción del curso. Más influencia que en las fases de entrega de materiales y de desarrollo de las actividades.

No obstante el trabajo de los profesores instructores (educativos) ha de realizarse con la participación de todos y en las cuatro líneas de desarrollo que hemos visto (Fig. 2):

- Bases teóricas del diseño.

- Práctica formativa (actividades de enseñanza y de aprendizaje).

- Investigación formativa y basada en el diseño.

- Diseño instruccional

En el documento del anexo distinguimos entre fases y líneas o componentes. Las fases son consecutivas en el tiempo. Las componentes operan en todo momento en el curso. En una fase tiene preminencia un tipo de componente, en la fase de desarrollo tiene preeminencia las actividades, la tutoría y la evaluación, pero son igualmente operativas las componentes de diseño, elaboración, teórica e investigativa, evaluación formativa y componente teórica. Y esto sucede con todo lo demás.

En el ANEXO 1 exponemos el procedimiento propuesto para diseñar un curso de este tipo, independientemente de que lo desarrollemos de forma completa y detallada en otros trabajos de naturaleza adecuada: artículos, guías, etc.

Fin de redacción del artículo: 18 de febrero de 2015. 
Zapata-Ros, M. (2015). El diseño instruccional de los MOOCs y el de los nuevos cursos abiertos personalizados. Revista de Educación a Distancia. 45(2). http://www.um.es/ead/red/45/zapata.pdf

\section{$\underline{\text { Referencias }}$}

Brandman, R. (2013) 5 Tips: Learn more effectively in class with Mastery Learning. Blog Coursera. http://blog.coursera.org/post/50352075945/5-tips-learn-moreeffectively-in-class-with

CIT (Center for Intructional Technologie) (2013) Building a Coursera Course Version 2.0 https://docs.google.com/document/d/1ST44i6fjoaRHvs5IWYXqJbiI31muJii_iqeJ_y1 pxG0/edit?pli=1

Clark, D. (2013). MOOCs: taxonomy of 8 types of MOOC. Donald Clark Plan B. http://donaldclarkplanb.blogspot.com.es/2013/04/moocs-taxonomy-of-8-types-ofmooc.html

Conole, G. (2013). MOOCs as disruptive technologies: strategies for enhancing the learner experience and quality of MOOCs. http://eprints.rclis.org/19388/

Crosslin, M (2014 May). Designing a Dual Layer cMOOC/xMOOC. http://www.edugeekjournal.com/2014/05/04/designing-a-dual-layer-cmoocxmooc/

Downes, S. (2011). Connectivism and Connective Knowledge 2011 (CCK11). How This Course Works. http://cck11.mooc.ca/how.htm

Fink, L.D. (2003), A Self-Directed Guide to Designing Courses for Significant Learning. http://www.deefinkandassociates.com/GuidetoCourseDesignAug05.pdf

Reigeluth, C. M., Merrill, M. D., Wilson, B. G., \& Spiller, R. T. (1980). The elaboration theory of instruction: A model for sequencing and synthesizing instruction. Instructional Science, 9(3), 195-219.

Reigeluth, C. M. \& Stein, R. (1983). Elaboration theory. Instructional design theories and models: An overview of their current status, Lawrence Erlbaum, Hillsdale, NJ, 335-381.

Reigeluth, C. M. (1999). The elaboration theory: Guidance for scope and sequence decisions. Instructional design theories and models: A new paradigm of instructional theory, 2, 425-453.

Reigeluth, C. M. (2012). Teoría instruccional y tecnología para el nuevo paradigma de la educación. RED, Revista de Educación a Distancia. Número 32.30 de septiembre de 2012. Consultado el (dd/mm/aaa) enhttp://www.um.es/ead/red/32

Siemens, G. (2014, May). Multiple pathways: Blending XMOOCs \& cMOOCs. http://www.elearnspace.org/blog/2014/05/06/multiple-pathways-blending-xmoocscmoocs/

Weller, M. (2013). Good post by Martin Weller [@mweller] on Coursera's new plans The Ed Techie: You can stop worrying about MOOCs 
now http://ow.ly/1WTQCf,http://nogoodreason.typepad.co.uk/no_good_reason/2013 /05/you-can-stop-worrying-about-moocs-now.html

Wiley, D. (2012). Thoughts on Conducting Research in MOOCs. http://opencontent.org/blog/archives/2205

Zapata-Ros, M. (2012). Teorías y modelos sobre el aprendizaje en entornos conectados y ubicuos. Bases para un nuevo modelo teórico a partir de una visión critica del conectivismo”. http://eprints.rclis.org/17463/

Zapata-Ros, M. (2013a). MOOCs, una visión crítica y una alternativa complementaria: La individualización del aprendizaje y de la ayuda pedagógica. Campus Virtuales, II, (1), 20-38.

Zapata-Ros, M. (2013b). Las teorías del aprendizaje y el diseño instruccional. El esquema incompleto. http://redesabiertas.blogspot.com.es/2013/04/las-teorias-delaprendizaje-y-el-diseno.html

Zapata-Ros, M. (2014a). Los MOOC en la crisis de la Educación Universitaria.: Docencia, diseño y aprendizaje. Amazon.

Zapata-Ros, M. (2014b). Charles Reigeluth: la personalización del aprendizaje y el nuevo paradigma de la educación para la sociedad postindustrial del conocimiento. En Carreras, J. S., \& Albert, M. E. (Eds.). Pensadores de ayer para problemas de hoy: teóricos de las ciencias sociales (Vol. 6) (pp. 153-191). Nau Llibres.

Zapata-Ros, M. (2015a). Sistemas de Apoyo al Aprendizaje y al Rendimiento. RED El aprendizaje en la Sociedad del Conocimiento. http://red.hypotheses.org/847

Zapata-Ros, M. (2015b). Diseño instruccional de cursos abiertos on-line. Universidad de Alcala. https://openeducation.blackboard.com/mooccatalog/courseDetails/view?course_id=_232_1 


\section{ANEXO 1}

\section{Guía y procedimiento para el diseño y la creación de un curso:}

\section{Objetivos y epítome.}

En esta parte hay que pensar sobre las expectativas que usted tiene como diseñador del curso, o las que tienen los diseñadores y promotores del curso. ¿Qué deben saber hacer los estudiantes para aprobar cada asignatura? Hay que pensar también qué concepto (epitome o síntesis de contenidos) da sentido global al curso y a la asignatura.

Cada una de las cosas que los alumnos deben saber o deben saber hacer constituyen los objetivos del curso y de las asignaturas. Y es lo que da sentido a todo lo demás. Los objetivos de conocimiento (conceptos y sistemas de ideas) y los objetivos de ejecución (competencias) debes de formularse de forma que sean evaluables, 0 de que se desglosen en otros objetivos evaluables. Igualmente deben formularse de manera que sea posible establecer formas de conseguirlos en sus distintos grados: Comprensión, adquisición y dominio. Por último los objetivos deben ser tales que posibiliten una secuencia de entrega de materiales que progresivamente acerquen a su consecución.

El epítome debe ser adecuado para que cada título de asignatura, de bloque o de unidad constituya un desglose del concepto global y signifique un acercamiento y una aportación en su consecución.

El sistema que constituye el curso es un sistema de entrega de materiales y de asistencia pautado y personalizado que tiene como referencia los objetivos y los criterios para ver si los alumnos cumplen con cada uno de ellos para pasar al siguiente en la secuencia. También debe der expresado de forma explícita y entendible por los alumnos lo que se espera que sean capaces de hacer y saber al final del curso para completarlo con éxito.

\section{Construcción de unidades.}

El curso debe ofrecer los contenidos y todo lo relacionado con ellos organizado en unidades. A priori hay una tradición que hace que la cantidad de contenidos 
de una unidad equivalga a lo que un alumno medio es capaz de desarrollar en uno o dos semanas de actividad. Esta organización ayuda a los alumnos a ubicarse dentro de un esquema de progreso en el desarrollo del curso. Sin embargo:

a) Por lo dicho en las justificaciones teóricas, la medida de la duración de una unidad es relativa y flexible. Relativa a la división de los objetivos en objetivos más simples, a las expectativas, de las que hemos hablado sobre competencias y dominio de tareas, sobre lo que un alumno puede trabajar y afianzar antes de pasar a la siguiente, y por último flexible en la duración.

b) $\mathrm{Y}$ debe tener coherencia interna, de forma que los contenidos equivalgan a la cantidad de contenidos que guarden relación con el título, o subepítome, que define la unidad.

Para construir las unidades utilizaremos las técnicas y herramientas de secuenciación de contenidos. Se han explicado qué son y cómo se aplican tanto las versiones clásicas como la versión primera de la Teoría de la Elaboración y la versión modificada (todas ellas de Reigeluth).

\section{CONFECCIÓN DE LA SECUENCIA \\ ELABORATIVA}

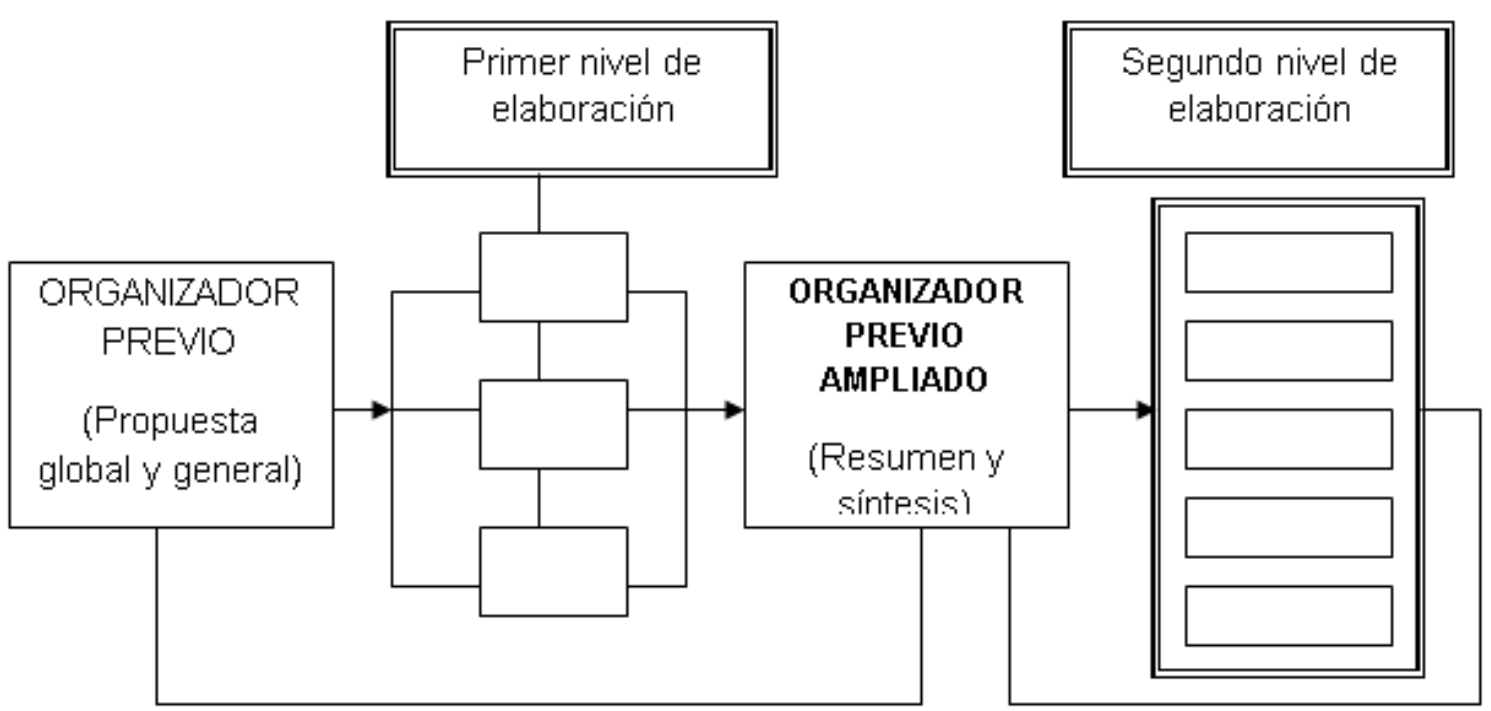

Fig. 1 Confección de la secuencia elaborativa

En esencia el resultado de aplicar estas técnicas nos da una progresión, de unidades didácticas, que constituye una secuencia. De manera que la organización de contenidos en un esquema plano de contenidos y de las 
relaciones que se establecen entre ellos (organizados en un mapa conceptual u otra representación) se transforma en unan lista lineal (Fig. 1). Y en fases sucesivas sucede lo mismo con las unidades didácticas y con los epígrafes (o con los bloques de contenidos inferiores, hasta llegar a la unidad mínima que es el concepto o la tarea elemental). En esta tarea debe de tenerse en cuenta el orden lógico, la secuencia procedimental, o el orden que determine la propia naturaleza disciplinar de los contenidos, pero sobre todo debe de pesar la propia capacidad psicológica de alumno de crear elementos de inserción de los nuevos elementos de conceptualización o de ejecución a partir de los existentes.



Fig. 2 Secuenciación: Transformación de una estructura ramificada en una estructura lineal

Es importante al crear unidades, que tengan una homogeneidad de extensión, podemos tener como referencia asignar a cada unidad lo que en condiciones normales un alumno medio puede trabajar en 1 semana $^{4}$.

4 En nuestra propuesta de personalización no hay una referencia clara y vinculante al tiempo. Cada unidad durará lo que dure para cada alumno. Esto significa que las unidades si bien tienen una duración estimativa, estarán siempre expuestas con una referencia clara a qué es preciso detrás de qué va, y para que contenidos sirve como condición necesaria. Es decir debe haber una inclusión en una secuencia. 
La unidad didáctica es además una unidad de evaluación y de autoevaluación. Si utilizamos la técnica de mastery learning, el alumno no deberá pasar a la unidad siguiente si no ha demostrado el dominio de las tareas que constituyen la unidad.

\section{Construir la guía docente de la unidad.}

Lo normal, e incluso lo indicado, es que el instructor, usted, desee incluir sus notas sobre cada unidad en un documento. Para poder utilizarlo en futuras ediciones, o para ésta, con el objetivo de tenerlo como referencia en las siguientes unidades. E incluso como medio de comunicación con sus colegas del mismo curso, de igual forma que a usted le gustará tener los documentos análogos de ellos para poder utilizar lo que ven, no repetir contenidos, ajustar sus enfoques con los otros profesores, y tenerlos presentes en las reuniones de coordinación docente. Estos documentos son las guías docentes.

En cada guía debe también, porque es útil, explicitar sus expectativas y los objetivos individuales de aprendizaje. Pero también cómo puede verificar si los estudiantes han logrado sus objetivos. Los criterios y métodos de evaluación. Los recursos que utiliza, comentando en qué ambiente y con qué ayuda los utiliza, incluyendo enlaces con las actividades - que es otro apartado imprescindible de la guía docente- y de aquellos y de estas con la evaluación y con los objetivos.

Es muy importante tener anotaciones sobre cómo hacer las evaluaciones para asegurar el dominio de las tareas y la consecución de los objetivos. Y con el progreso general en la asignatura.

Incluir criterios de evaluación detallados, que nos indiquen la progresión en la ejecución de las tareas para llegar al dominio en al menos tres niveles: Comprensión, asimilación, dominio, así como criterios para asegurar la consecución de cada uno.

También los que se examinen o se sometan al mastery learning lo podrán hacer en cualquier momento, o no hacer. 


\section{Guía didáctica de la unidad. Describir lo que el alumno debe hacer para que se sienta guiado. Se puede escribir cada unidad en una hoja de cálculo.}

La guía didáctica de la unidad es el principal documento para que el alumno sepa en qué lugar está en cada momento en su progresión hacia los objetivos.

Usted debe de tener claro esto de manera que el lenguaje sea adecuado al tipo de alumnos que van a seguir el curso y no tenga tecnicismos de tipo pedagógico o de otra naturaleza distinta de la materia que el alumno va a aprender, o de las metáforas o imágenes que necesita para una mayor comprensión, y aún éstas deben de estar escritas en un estilo neutro y no local, para posibilitar la comprensión de los alumnos en los lugares más diversos.

El formato de la guía puede ser secuencial, es decir conteniendo una secuencia de elementos menores de contenidos, tareas o elaboraciones (ítems o epígrafes). Cada uno con sus actividades, recursos, evaluación, etc. O puede seguir un modelo de dos dimensiones, tabla, matriz utilizando una hoja de cálculo. Le recomendamos esta opción, aunque yo personalmente he utilizado durante bastante tiempo la primera.

Si utilizamos este modelo tenemos que tener claras dos cosas:

- La secuencia de epígrafes (elementos de contenidos, tareas o elaboraciones) de manera que cada una constituya una fila o línea en la tabla.

- Las dimensiones que constituyen la unidad didáctica: Título, nombre o síntesis del contenido o tareas; descripción de los contenidos; objetivos, tareas o competencias que se deben dominar; actividades (lecturas, ver vídeos, realizar síntesis o trabajos, etc.); recursos y sistema de evaluación. De todas formas esto lo describiremos con más detalle después. Lo importante es que estas dimensiones constituyan las columnas de la tabla.

En cualquier caso cada guía de unidad debe contener referenciado de forma clara el material didáctico (vídeos, lecturas, evaluaciones) que guiarán a los estudiantes hacia la objetivos de aprendizaje de la unidad. Las unidades incluirán además elementos de evaluación graduados y progresivos y, en su caso, diversificados, con base a los materiales entregados en la unidad. Ofrecemos un ejemplo de hoja de cálculo desarrollada con un caso 
general y fácilmente comprensible en el ANEXO 2 y en una hoja de cálculo de Google drive 5 .

Describimos ahora con más detalle los elementos de que consta la unidad como columnas de la hoja de cálculo que utilizamos como guía didáctica:

- Título y fecha de la edición de la guía, así como versión

El título debe reflejar una síntesis de los contenidos (epitome) o un concepto globalizador donde se inserten los ocntenidos, o el objetivo más importante. Pero es preferible lo primero.

- Título del epígrafe. Nombre o síntesis del contenido o tareas; descripción de los contenidos, primer o segundo nivel de elaboración

En una versión normal de MOOC, o de curso estándar, nos conformaríamos con un primer nivel de elaboración, que nos da los temas de que consta la unidad (ver el ejemplo de guía de unidad de la asignatura $\mathrm{SIC}^{5}$ en el ANEXO 2). Pero en un curso personalizado o en un trabajo bien hecho, o simplemente si deseamos desglosar los contenidos o tareas para el método de Mastery learning, tendríamos que llegar al segundo, tercer o cuarto nivel de elaboración, que ya nos daría las claves del contenido, competencias o tareas que habría que dominar. Ver el ejemplo de secuencia elaborativa de Programación Lineal ${ }^{6}$ en el ANEXO 3.

\section{- Objetivos de Aprendizaje}

Qué van a aprender los estudiantes en la unidad. No tienen porqué corresponderse las celdas de epígrafes con las celdas de objetivos. Un epígrafe puede contribuir parcialmente a un objetivo (sobre todo si es procedimental, de ejecución o una tarea). Incluso un único epígrafe puede desarrollar actividades para varios objetivos.

\footnotetext{
${ }^{5}$ La hoja de cálculo está en la dirección

https://docs.google.com/spreadsheet/ccc?key=0AjcfGEtQELYVdHFGRE5JeVdLWTFabXBSSTh4dzE3amc\& usp=sharing

${ }^{6}$ https://docs.google.com/document/d/1DxF8JGA2a-G0-4FA9IfJjYVq6VUIbp4XKO6Do-iK3e4/edit?pli=1
} 
- Tareas o competencias que se deben dominar; (sólo para el caso de Mastery learning y para cursos personalizados)

Expresión muy sucinta de lo que debe saber hacer para pasar a la unidad siguiente. Debe ser verificado por el profesor-alumno asistente que tenga asignado.

- Actividades (lecturas, participar en los foros, visitar webs, ver vídeos, realizar síntesis o trabajos, etc.)

Actividades que se proponen para ayudar a alcanzar los objetivos. Son objeto de autoevaluación o de apoyo por los profesores asistentes en el caso de cursos personalizados.

\section{- Autoevaluación}

En este apartado se indica el procedimiento, su enlace en caso de que esté en la web para que el alumno realice la autoevaluación de las actividades propuestas.

- Evaluaciones por dominio de tarea sólo para el caso de Mastery learning y para cursos personalizados.

Es preciso haber realizados antes una secuenciación utilizando alguna de las técnicas clásicas (Análisis de contenidos, análisis de las tareas, Teoría de la Elaboración). En el caso de la Teoría de la elaboración habría que realizar reelaboraciones hasta conseguir un nivel adecuado para aplicar el Mastery Learning.

En todo caso hay hacer una indicación sucinta de los criterios que el profesor va a tener en cuenta para aceptar que se ha producido el nivel suficiente de

\section{Comprensión.}

Tareas, preguntas conceptuales, intervenciones en el foro, etc. que en el desarrollo de las actividades el alumno tendrá que hacer o responder para asegurar la comprensión. El PA tomará notas y devolverá información textual o verbal. 


\section{Asimilación. Incorporación. Atribución de significado}

Los alumnos consiguen realizas las tareas de forma autónoma, o resolver los problemas sin ayuda.

\section{Dominio}

Los alumnos resuelven todos los problemas y realizan todas las tareas de forma autónoma en todos los casos y en situaciones y con datos distintos.

- Recursos. Lista de materiales a utilizar para este epígrafe y para realizar las actividades de que consta: Documentos (PDF, doc, etc), videos temáticos, vídeos de conferencias, otros vídeos, lecturas en documento o libro impreso, PPTs, enlaces web, foros, etc.

Tenga en cuenta las necesidades de los alumnos para utilizar el recurso y haga cuantas anotaciones sean necesarias en este sentido, o enlace con una guía particular para la actividad utilizando este recurso. Por ejemplo: De qué forma deberían seguir estos videos con el fin de ayudar a aprender más efectivamente.

Es muy importante contar con los derechos de autor. No incluya materiales delos que no disponga de los derechos de copia. Utilice preferentemente recursos abiertos (http://search.creativecommons.org/) o cree recursos propios. Con las facilidades de Internet y de edición es posible

- Propuestas de Evaluaciones alternativas o complementarias (Deje abiertas otras posibilidades para que los estudiantes demuestren que han aprendido o que saben hacer)

Para ello debe dejar bien claro las cuestiones o temas que los alumnos deben saber o deben saber hacer.

\section{Crear y organizar materiales para cada unidad.}

No es una buena idea ni un buen sistema de diseño primero disponer de recursos y luego organizar las actividades y el contenido en torno a ellos. Eso es una mala práctica. 
La idea es justo la contraria. El buen diseñador tiene una idea clara de cuáles son los objetivos, de qué actividades contribuyen mejor a ellos, de si esas actividades son evaluables y cómo. A partir de ahí su labor es crear-buscarconocer recursos y organizarlos en un esquema de secuenciación y dificultad progresiva, de forma que no haya saltos, discontinuidades, ni vacíos cognitivos.

Tampoco valen muchas ideas y patrones de la enseñanza presencial, o de la enseñanza online local, parcial o limitada. En ella podemos recurrir a imágenes, metáforas o expresiones locales o con limitaciones de comprensión que luego pueden ser subsanadas o completadas en conferencias, clases presenciales 0 tutorías.

Todo tiene que ser pues explícito y con un lenguaje estandarizado sin referencias a localismos o a la apelación o la sugerencia a chistes, a personajes o a situaciones supuestamente conocidos por todos. Ni tampoco a ideas morales, políticas o religiosas, ni a costumbres supuestamente aceptadas por todos.

No establezca el compromiso de fechas sino de tiempos establecidos $\mathrm{y} / \mathrm{o}$ negociados para la ejecución de tareas. Respetar ese compromiso, teniendo en cuenta que los estudiantes pueden realizar lecturas y ejecuciones en distintos ritmos y horarios, y ver vídeos 2 ó 3 veces.

Es muy importante para la metodología Mastery learning disponer de muchas pruebas, preguntas y tareas de naturaleza y dificultad similar, para evitar el efecto de la asincronía y de la repetición de las pruebas sin que se repitan los ejercicios.

Se recomienda el siguiente procedimiento para la creación de materiales didácticos:

\subsection{Diseño de evaluaciones}

- Diseñe la evaluación, incluso preparando las actividades, tareas y preguntas de evaluación antes de la organizar los recursos, particularmente de elegir los vídeo y de escribir el guión de los foros. De esta forma habrá una correspondencia entre estos y aquella.

- Formule las preguntas de evaluación y otras pruebas pensando y teniendo presente los los objetivos de aprendizaje. 
- Cree muchas versiones y variaciones de las tareas, ejercicios y cuestionarios para facilitar el aprendizaje de los estudiantes y su evaluación, ya que retomarán muchas veces las tareas y las evaluaciones. Sería muy bueno estar coordinado y tener una metodología efectiva de trabajo en colaboración con los informáticos para que hubiesen unas buenas bases de datos de actividades y de pruebas parametrizadas por niveles de dominio, dificultad, etc. y que pueden obtener de forma aleatoria sin repetición ni sesgo.

- Considere, y tenga el mismo talante con los informáticos, para la disposición y para el uso de la herramienta de autoevaluación por los estudiantes y para que los TA evalúen y tutoricen el trabajo de los alumnos sobre la base de una rúbrica que el sistema proporcione.

- Por último piense en la frustración y en el abandono que se deriva de un nivel inadecuado de los ejercicios, tareas, pruebas y preguntas. No debe haber saltos cognitivos. Vigile este punto. Debe de asegurarse también la continuidad y la progresión en vídeos y en lecturas. No debe dar por supuesto nada, y debe decir al principio de la actividad qué conocimientos previos se suponen.

\subsection{Establecer foros}

- Los foros fomentan la discusión y ponen en marcha mecanismos de búsqueda y de elaboración de argumentos y de pruebas. También favorecen que los alumnos compartan sus hallazgos y resolución de problemas.

- Una vez que haya decidido utilizar los foros, lo conveniente es hacer uno por unidad. Cree un guion teniendo en cuenta el tema de unidad, los recursos y actividades propuestos en la guía y piense en la evaluación.

- Cuando publique el guión pida la participación en el debate de forma explícita y dé las normas.

- Iniciar un nuevo foro un tiempo después de iniciar la unidad o de publicar la guía de la unidad. Igualmente déjelo abierto unos días o unas semanas después de concluir oficialmente la unidad. $O$ déjelo abierto permanentemente.

- Una buena moderación es imprescindible. Sea estricto con la llamada al tema, no permita los off topics. Elija siempre que pueda la opción de mensajes encadenados por el asunto, y que el contenido sea siempre relativo al asunto. Para compensar cree un foro libre, el que desde hace tiempo [enlace] algunos venimos Ilamando "cafetería"

- Los foros llevan mecanismos de encadenamiento de las intervenciones, pero las redes sociales también y los alumnos ya las conocen. Además 
hay complejas y completas herramientas de la web social. Si la conoce es preferible que utilice estos entornos. Llevan videoconferencias y videogrupos, discos virtuales y herramientas de trabajo y de edición compartido. Los estudiantes se benefician de una gran cantidad de ayuda que reciben de otros alumnos de la clase. Son preferibles a los foros simples. Si se encuentra cómodo pues, son preferibles los entornos sociales.

\subsection{Compilar materiales para el curso (PDF y PPTs).}

- Si va a utilizar materiales con derechos de autor debe contar con las correspondientes licencias y permisos. Si no es así considere la búsqueda de alternativas, gestionar de nuevo los permisos, utilizar materiales de licencia CC o de Open Access, o bien cree sus propios materiales.

- Los materiales deben de estar referenciados con el orden en que se van a utilizar asociados a unos objetivos y a unas actividades en la guía didáctica. Deben reseñarse, las páginas o transparencias que se van a utilizar en concreto.

\subsection{Creación de videos.}

- Plantéese como objetivo que cada vídeo tenga una duración de aproximada de 5 minutos (como máximo10), y que el visionado ocupe en torno a 1 ó 2 horas dedicadas a vídeos en total por semana.

- Inserte en la guía, o en el propio vídeo, 1 a 3 preguntas como autoevaluación

- Introduzca en el propio vídeo, o en la guía, scripts de esquema y temas de debate.

- Si va a crear sui propio vídeo piense y elija el formato más adecuado para el contenido que va a tratar: diapositivas comentadas, "busto parlante", etc.

- Utilice el vídeo con prudencia, consume mucho tiempo, sólo para cuestiones que tengan especial dificultad conceptual o para resolución de problemas que entrañen aspectos gráficos explicados, esquemas, etc. Utilice estilos directos como de clase presencial.

- En el transcurso de la unidad, puede crear y publicar vídeos breves ad hoc para complementar las enseñanzas y las dificultades surgidas, y manifestadas en tutoría o en los foros, "en tiempo real". 


\subsection{Organizar videoconferencias y videogrupos (hangout)}

- La Video Conferencia o Video Clase sustituye a la clase expositiva presencial y adopta su formato y su metodología. Únicamente a diferencia de aquella hay que cuidar el tema y el contenido, para que sea relevante (sea clave para otros contenidos y para otroa aprendizajes) y trate las cuestiones de especial dificultad conceptual que no puedan abordarse en otras actividades.

- Una video conferencia hay que dividirla en trozos relativamente pequeños en los que se intercales resúmenes y preguntas que apelen a la autoevaluación.

- El videogrupo sustituye a la sesión presencial tradicional de la enseñanza a distancia, en el sentido de que supone una actividad de ajuste y de regulación para evitar que alumnos, o bloques de materia, se queden descolgados de la marcha general del curso. Es importante prepararlos con cuestionarios previos o simplemente haciendo llamadas a cuestiones en los foros. En los videogrupos se ha de propiciar la interacción entre los alumnos, posibilitando que planteen sus dudas y que sean respondidas por otros estudiantes con sus propias palabras tal como lo han comprendido.

En cualquier caso los estudiantes valoran especialmente dos características de los materiales:

- La interactividad: que tengan respuestas adecuadas a distintas situaciones.

- Que propicien el pensar en el tema de nuevas maneras

6. Los Profesores Asistentes. Establezca criterios claros acerca de la comunicación con los alumnos. Evite falsas expectativas.

Como hemos visto hay unos únicos profesores instructores que deben participar y tener competencias en todas las funciones educativas de las asignaturas. Son los especialistas en la materia, en los recursos, en su organización, en la enseñanza (estrategias docentes, actividades, objetivos y evaluación), deben conocer a los alumnos en la medida de lo posible e interactuar entre ellos. Cualquier división de esta figura y en su responsabilidad supone una fragmentación en el sistema pedagógico con el consiguiente deterioro de la calidad. Para decir esto nos basamos en el problema 2 sigma y 
en otras investigaciones que validan las teorías en las que nos basamos, descritas al principio.

Sin embargo la metodología docente de Mastery learning supone la existencia de unos Profesores Asistentes o Asistentes en la Enseñanza (en todo caso asistentes del alumno, no del profesor. Si bien actúan coordinados y supervisados por él y bajo su responsabilidad). En general se les conoce con las siglas TA (de Teaching Assinstant). Estos profesores verifican el progreso, hasta el dominio, de la tarea de los alumnos.

Antes de la organización del curso, pero sobre todo antes del inicio del curso, los instructores deben determinar las funciones de los ayudantes del curso. Tanto para los alumnos implicados en el Mastery learning como para el resto.

La función del TA en el dominio de la tarea es clave y su responsabilidad máxima. Deben asegurar que cada alumno supera cada nivel para pasar al siguiente, deben atenderlos en las dificultades, proponer ritmos distintos y problemas o tareas alternativas, y hacerlo de forma flexible, personal e interactuando con ellos. En definitiva deben de ser profesores especializados en esta tarea cuya complejidad no se debe menospreciar.

El TA también puede ayudar a los alumnos no implicados en el Mastey learning, alumnos a los que podemos llamar libres. Pero en este caso el papel de TA lo pueden cumplir otros alumnos (alumnos asistentes, Student Assistant SA o Learner Assistant LA) y también deben estar coordinados por el instructor.

Todos ellos deben de coordinarse para supervisar el material que producen los estudiantes en los foros y en la web social: Mensajes, post, comentarios, etc. detectando de forma temprana posibles déficits o frustraciones.

Tenga en cuenta que este monitoreo proporciona una inestimable retroalimentación.

Se ha de dejar claro que el instructor no puede atender todas las interacciones, Los TA cumplen esta función como la de filtro para las interacciones que deba atender el instructor.

Los alumnos aceptan esto con satisfacción. 
El instructor debe comunicar expresamente a los estudiantes sobre el papel que va a desempeñar en las actividades. No debe crear expectativas de atender a todos en todo. Por ejemplo, los estudiantes no deben esperar que el instructor responda todos los correos electrónicos.

Además de expresarlo de forma directa, preferiblemente con un vídeo del instructor, sería bueno incluirlo de forma razonada y afectuosa en una declaración inicial. Y desde luego debe figurar en las normas del curso.

No obstante los alumnos se sienten bien si se sienten conectados de alguna forma con el instructor, por tanto éste debería con cierta frecuencia hacer intervenciones en los foros y enviar algunos mensajes especialmente con motivo de alguna intervención relevante o representativa de una duda generalizada, de algún alumno. Responder a mensajes en el foro es una buena manera de construir un sentido de comunidad y proporciona seguridad, profundidad y visión más allá de lo que se imparte, además de ahuyentar la banalidad, tan peligrosa.

Es importante también la función orientadora. Tanto el instructor como los TA deben de introducir de forma frecuente en los foros y en todas las posibilidades de interacción que tengan, referencias a los criterios de evaluación y en general a todo aquello que favorezca la consecución de los objetivos de aprendizaje y a la obtención de la acreditación o de la insignia. 
ANEXO 2.-

\begin{tabular}{|c|c|c|c|c|c|c|c|c|c|c|}
\hline & $\begin{array}{l}\text { Título de la } \\
\text { unidad: }\end{array}$ & $\begin{array}{l}\text { Metodologías de } \\
\text { investigación y } \\
\text { nueva naturaleza } \\
\text { de la ciencia en la } \\
\text { SIC. }\end{array}$ & \begin{tabular}{|l} 
Asignatura: \\
Sociedad de la \\
Información y \\
del \\
Conocimiento
\end{tabular} & & & & & & & \\
\hline & Estudios: & $\begin{array}{l}\text { Máster } \\
\text { universitario. } \\
\text { Segundo curso. }\end{array}$ & & & & & & & & \\
\hline & & & & & \multicolumn{3}{|c|}{ Evaluaciones por dominio de tarea } & & & \\
\hline \multirow[t]{2}{*}{$\begin{array}{l}\text { Título. } \\
\text { Nombre o } \\
\text { síntesis del } \\
\text { contenido o } \\
\text { tareas; } \\
\text { descripción } \\
\text { de los } \\
\text { contenidos, } \\
\text { primer o } \\
\text { segundo } \\
\text { nivel de } \\
\text { elaboración }\end{array}$} & $\begin{array}{l}\text { Resultado de } \\
\text { aprendizaje. } \\
\text { Objetivos de } \\
\text { aprendizaje. }\end{array}$ & $\begin{array}{l}\text { Tareas o competencias que } \\
\text { se deben dominar }\end{array}$ & $\begin{array}{l}\text { Actividades } \\
\text { (lecturas, ver vídeos, } \\
\text { realizar síntesis o } \\
\text { trabajos, etc.) }\end{array}$ & $\begin{array}{l}\text { Autoeva- } \\
\text { luación }\end{array}$ & $\begin{array}{l}1 . \\
\text { Comprensión }\end{array}$ & $\begin{array}{l}\text { 2. Asimilación. } \\
\text { Incorporación. } \\
\text { Atribución de } \\
\text { significado }\end{array}$ & 3. Dominio & Recursos & Ubicación & $\begin{array}{l}\text { Intervenciones } \\
\text { significativas en el fo } \\
\text { (min.) }\end{array}$ \\
\hline & & & & & sí & sí & no & & & \\
\hline
\end{tabular}




\begin{tabular}{|c|c|c|c|c|c|c|c|c|c|}
\hline $\begin{array}{l}\text { Bienvenida y } \\
\text { presentación }\end{array}$ & \begin{tabular}{|l} 
Bienvenida \\
general para \\
los estudiantes. \\
Conocer la \\
información y \\
normas de la \\
asignatura y \\
presentación \\
de los \\
componentes.
\end{tabular} & $\begin{array}{l}\text { Conocerse personalmente y } \\
\text { conocer sus expectativas. }\end{array}$ & & & & & & & 1 \\
\hline \begin{tabular}{|l|} 
La \\
investigación \\
formativa en \\
el contexto \\
de la \\
investigación \\
basada en el \\
diseño \\
\end{tabular} & $\begin{array}{l}\text { Distinguir las } \\
\text { metodologias } \\
\text { de } \\
\text { investigación } \\
\text { basada en el } \\
\text { diseño de las } \\
\text { metodologías } \\
\text { clásicas de } \\
\text { investigación. } \\
\text { Y distinguir de } \\
\text { entre aquéllas } \\
\text { las } \\
\text { metodologías } \\
\text { de } \\
\text { investigación } \\
\text { formativa } \\
\end{array}$ & $\begin{array}{l}\text { Analizar un proyecto de } \\
\text { investigación y justificar, } \\
\text { señalando los rasgos o } \\
\text { carcaterísticas de él en las } \\
\text { que se basan, si se trata de un } \\
\text { diseño experimental, de una } \\
\text { investigación descriptiva o } \\
\text { de una investigación basada } \\
\text { en el diseño. Analizar un } \\
\text { proyecto de investigación } \\
\text { formativa señalando los } \\
\text { rasgos que lo caracterizan } \\
\text { como tal. }\end{array}$ & $\begin{array}{l}\text { Lectura de "La } \\
\text { investigación } \\
\text { formativa y la } \\
\text { investigación basada } \\
\text { en el diseño: Dos } \\
\text { perspectivas de } \\
\text { alcance", Zapata- } \\
\text { Ros, M. (2010). Ver } \\
\text { vídeo e intervenir en } \\
\text { el foro. }\end{array}$ & $\begin{array}{l}\text { Realizaer } \\
\text { el módulo } \\
\text { de } \\
\text { autoevalua } \\
\text { ción } \\
\text { "IBD-IF" }\end{array}$ & $\begin{array}{l}\text { Cuestionario } \\
\text { de preguntas } \\
\text { "Evaluación } \\
\text { IBD-IF } \\
\text { Comprensión" }\end{array}$ & $\begin{array}{l}\text { Cuestionario de } \\
\text { preguntas } \\
\text { "Evaluación IBD- } \\
\text { IF Incorporación" }\end{array}$ & $\begin{array}{l}\text { Cuestionario } \\
\text { de preguntas } \\
\text { "Evaluación } \\
\text { IBD-IF } \\
\text { Dominio" }\end{array}$ & \begin{tabular}{|l|} 
RED - Revista de \\
Educación a Distancia. \\
Número 22.15 de mayo \\
de 2010. Consultado el \\
[dd/mm/aaaa] en \\
http://www.um.es/ead/red/ \\
22
\end{tabular} & 2 \\
\hline
\end{tabular}




\begin{tabular}{|c|c|c|c|c|c|}
\hline $\begin{array}{l}\text { Investigación } \\
\text { formativa }\end{array}$ & \begin{tabular}{|l|} 
Lectura de Págs. 181 \\
a 200 de Diseño de la \\
instrucción.Teorías y \\
modelos. Reigeluth, \\
C. M. Ver vídeo e \\
intervenir en el foro.
\end{tabular} & ... preguntas & ... preguntas & ... preguntas & $\begin{array}{l}\text { Reigeluth, C. M. (Ed.) } \\
\text { (2000). Diseño de la } \\
\text { instrucción.Teorías y } \\
\text { modelos.Vol. I y II. } \\
\text { Madrid. Aula XXI, } \\
\text { Santillana. Parte II. 8. } \\
\text { Investigación formativa. } \\
\text { Original: Reigeluth, C. M. } \\
\text { \& Frick, T. W. (1999). } \\
\text { Formative research: A } \\
\text { methodology for creating } \\
\text { and improving design } \\
\text { theories. In C. M. } \\
\text { Reigeluth (Ed.), } \\
\text { Instructional design } \\
\text { theories and models: A } \\
\text { new paradigm of } \\
\text { instructional theory. (pp. } \\
\text { 5-29). Hillsdale, NJ: } \\
\text { Lawrence Erlbaum } \\
\text { Associates } \\
\end{array}$ \\
\hline $\begin{array}{l}\text { Investigación } \\
\text { basada en el } \\
\text { diseño }\end{array}$ & $\begin{array}{l}\text { Lectura de Rinaudo, } \\
\text { M.C. y Donolo, D. } \\
\text { (2010). Estudios de } \\
\text { diseño. Una } \\
\text { perspectiva } \\
\text { promisoria en la } \\
\text { investigación } \\
\text { educativa. Ver vídeo } \\
\text { e intervenir en el } \\
\text { foro. }\end{array}$ & ... preguntas & ... preguntas & ... preguntas & $\begin{array}{l}\text { Rinaudo, M.C. y Donolo, } \\
\text { D. (2010). Estudios de } \\
\text { diseño. Una perspectiva } \\
\text { promisoria en la } \\
\text { investigación educativa. } \\
\text { RED - Revista de } \\
\text { Educación a Distancia. } \\
\text { Número } 22.15 \text { de mayo } \\
\text { de } 2010 . \text { Consultado el } \\
\text { [dd/mm/aaaa] en } \\
\text { http://www.um.es/ead/red/ } \\
22 \\
\end{array}$ \\
\hline
\end{tabular}




\begin{tabular}{|c|c|c|c|c|c|}
\hline \begin{tabular}{|l|} 
Retos y \\
tendencias \\
en el futuro \\
de la \\
investigación \\
del \\
aprendizaje \\
con \\
tecnologías \\
digitales. \\
\end{tabular} & $\begin{array}{l}\text { Lectura de "Retos y } \\
\text { tendencias sobre el } \\
\text { futuro de la } \\
\text { investigación acerca } \\
\text { del aprendizaje con } \\
\text { tecnologías } \\
\text { digitales". Begoña } \\
\text { Gros. Ver vídeo e } \\
\text { intervenir en el foro. }\end{array}$ & ... preguntas & ... preguntas & ... preguntas & $\begin{array}{l}\text { Retos y tendencias sobre } \\
\text { el futuro de la } \\
\text { investigación acerca del } \\
\text { aprendizaje con } \\
\text { tecnologías digitales. } \\
\text { Begoña Gros. RED. } \\
\text { Revista de Educación a } \\
\text { Distancia. Número 32 } \\
\text { http://www.um.es/ead/red/ } \\
\text { 32 } \\
\end{array}$ \\
\hline $\begin{array}{l}\text { La ciencia } \\
\text { compartida. } \\
\text { E-ciencia }\end{array}$ & $\begin{array}{l}\text { Lectura de La } \\
\text { investigación y la } \\
\text { edición científica en } \\
\text { la web social: La } \\
\text { ciencia compartida. } \\
\text { Miguel Zapata Ros. } \\
\text { Ver vídeo e } \\
\text { intervenir en el foro. }\end{array}$ & ... preguntas & ... preguntas & ... preguntas & $\begin{array}{l}\text { La investigación y la } \\
\text { edición científica en la } \\
\text { web social: La ciencia } \\
\text { compartida. Miguel } \\
\text { Zapata Ros.RED. } \\
\text { Docencia universitaria en } \\
\text { la Sociedad del } \\
\text { Conocimiento, núm. } 3 \\
\text { www.um.es/ead/reddusc/3 } \\
\text { / }\end{array}$ \\
\hline
\end{tabular}


ANEXO 3.- Resultado de la confección una la secuencia elaborativa.

\begin{tabular}{|c|c|c|c|c|}
\hline $\begin{array}{l}\text { Organizador } \\
\text { previo }\end{array}$ & $\begin{array}{l}\text { Primer nivel de } \\
\text { elaboración }\end{array}$ & $\begin{array}{l}\text { Segundo nivel de } \\
\text { elaboración }\end{array}$ & $\begin{array}{l}\text { Tercer nivel de } \\
\text { elaboración }\end{array}$ & $\begin{array}{l}\text { Cuarto nivel de } \\
\text { elaboración }\end{array}$ \\
\hline \multirow[t]{7}{*}{$\begin{array}{l}\text { El problema de } \\
\text { Programación } \\
\text { Lineal }\end{array}$} & \multirow[t]{6}{*}{ Planteamiento } & \multirow[t]{5}{*}{ Función de beneficios } & $\begin{array}{l}\text { Planteamiento: Técnicas de } \\
\text { resolución de problemas. }\end{array}$ & $\begin{array}{l}\text { Identificar en el enunciado } \\
\text { unidades como variables, costes } \\
\text { por unidad como coeficientes, y } \\
\text { enunciar la función de beneficio } \\
\text { como función lineal de dos } \\
\text { variables }\end{array}$ \\
\hline & & & \multirow[t]{4}{*}{ Funciones lineales } & $\begin{array}{l}\text { Parámetros de las funciones } \\
\text { lineales, pendiente. }\end{array}$ \\
\hline & & & & $\begin{array}{l}\text { Identificar la recta de nivel con la } \\
\text { función beneficio. }\end{array}$ \\
\hline & & & & Rectas en el plano \\
\hline & & & & $\begin{array}{l}\text { Rectas de nivel sentido de la } \\
\text { traslación paralela. }\end{array}$ \\
\hline & & Conjunto de restricciones & $\begin{array}{l}\text { Inecuaciones lineales de dos } \\
\text { incógnitas. }\end{array}$ & $\begin{array}{l}\text { Sentido, interpretación. Las } \\
\text { soluciones gráficas del sistema } \\
\text { como soluciones posibles del } \\
\text { problema de programación lineal }\end{array}$ \\
\hline & Resolución & Conjunto de soluciones & Polígono de soluciones & $\begin{array}{l}\text { Obtener el polígono de } \\
\text { soluciones e identificarlo con el } \\
\text { conjunto de soluciones. }\end{array}$ \\
\hline
\end{tabular}

El diseño instruccional de los MOOC y el de los nuevos cursos abiertos personalizados. 


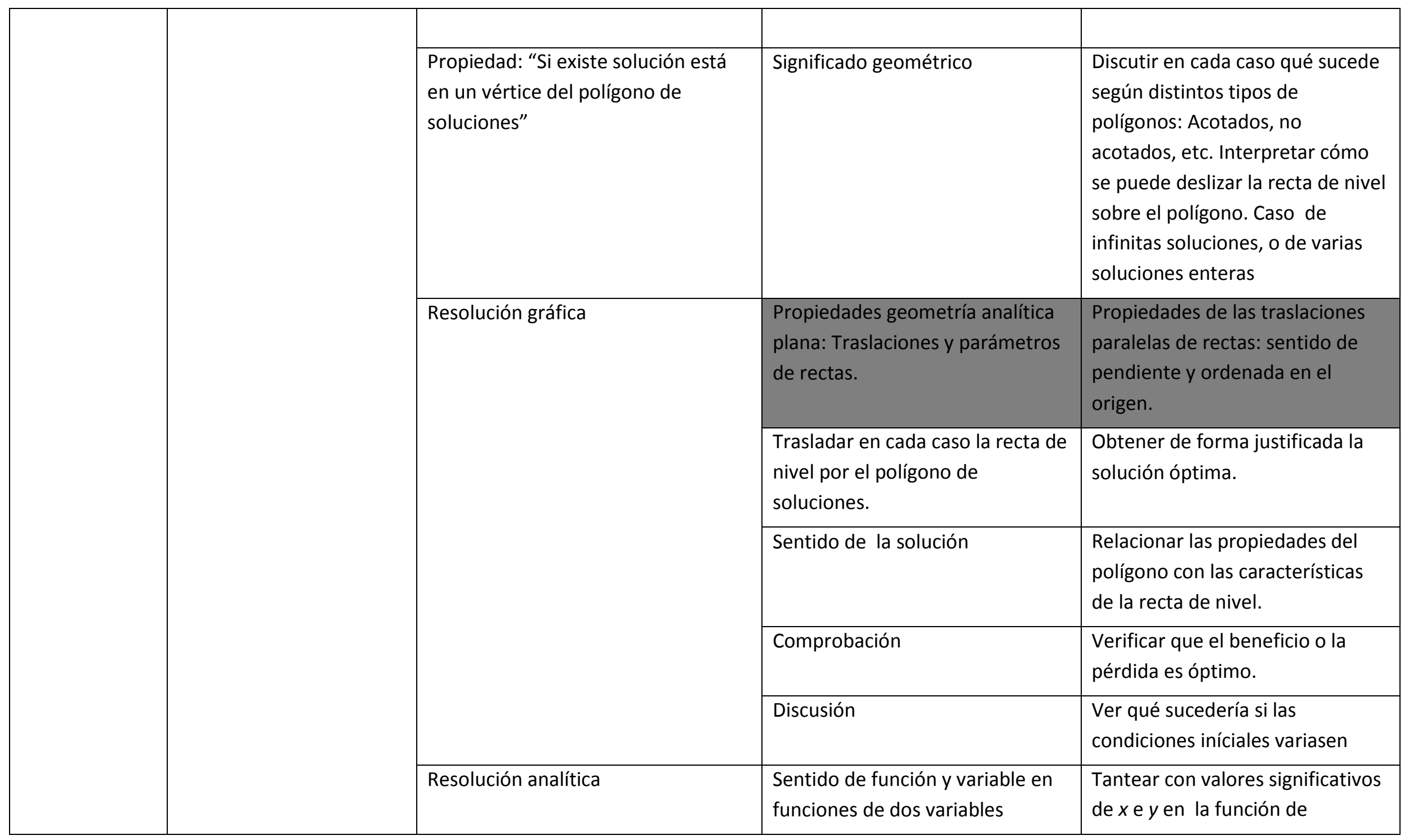

El diseño instruccional de los MOOC y el de los nuevos cursos abiertos personalizados. 


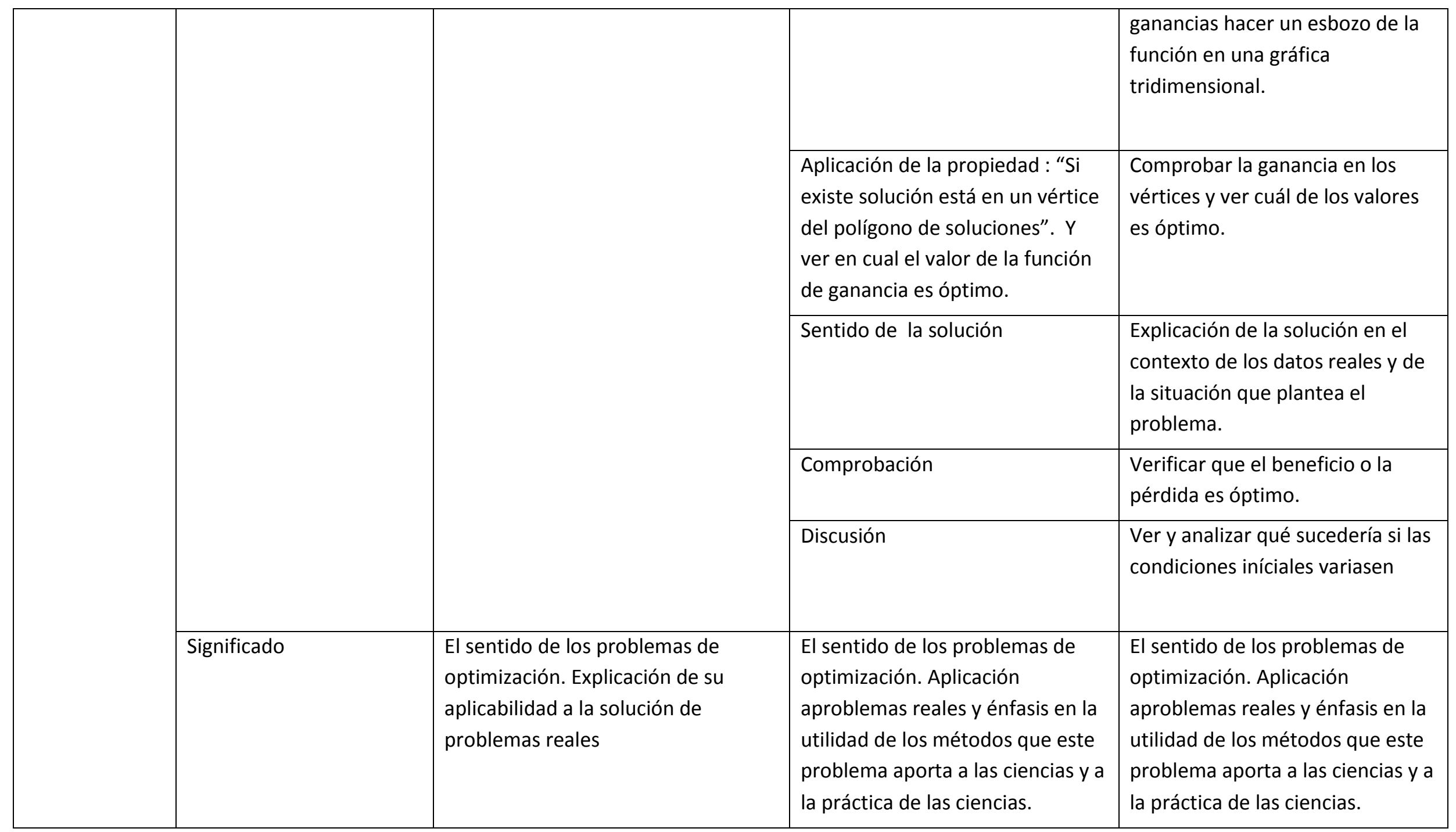




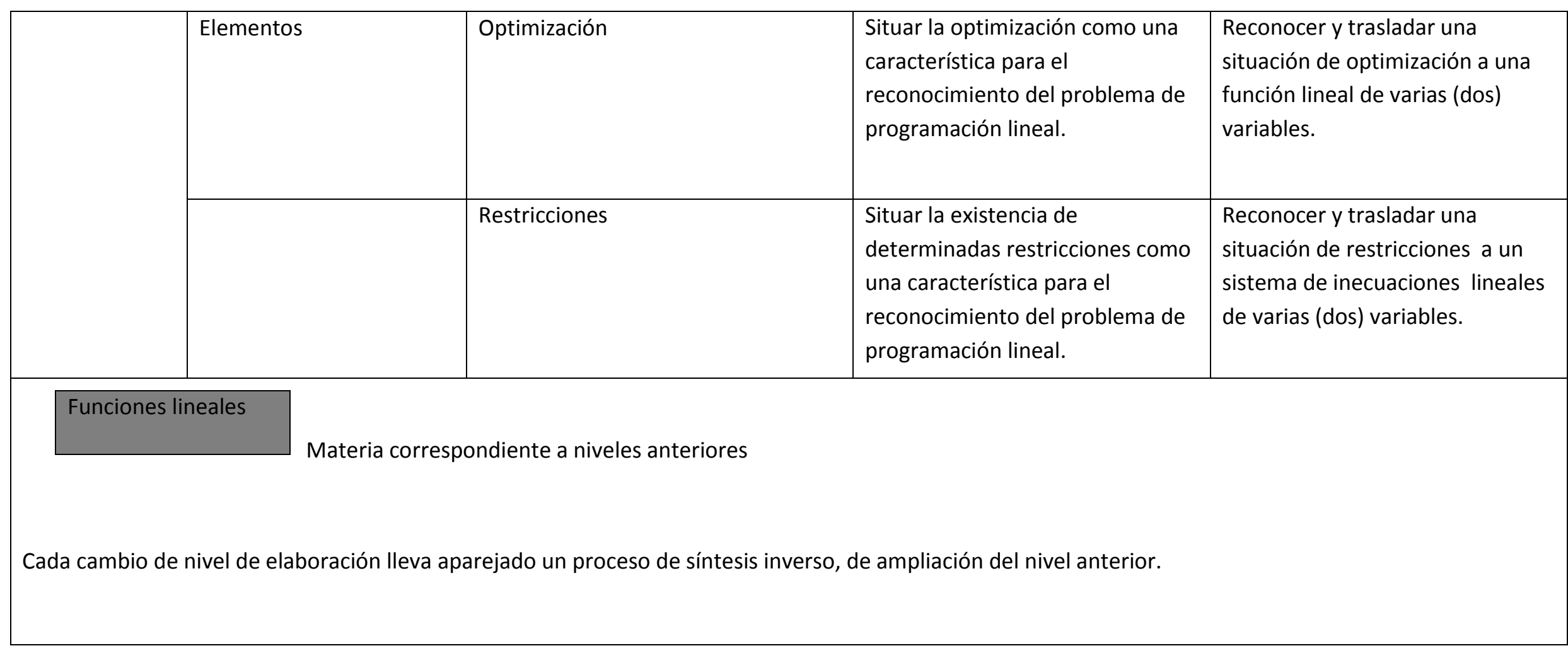

